\title{
Abdominal cystic lymphangioma mimicking ovarian mass: A case report and literature review
}

\author{
ALIKI TYMPA $^{1}$, CHARALAMPOS GRIGORIADIS ${ }^{2}$, KASSIANI THEODORAKI $^{1}$ and IOANNIS VASSILIOU $^{3}$ \\ ${ }^{1}$ First Department of Anesthesiology, Aretaieion Hospital, Medical School, University of Athens, Athens 11528; \\ ${ }^{2}$ Department of Obstetrics and Gynecology, Leto Maternity Hospital, Athens 11524; ${ }^{3}$ Second Department of Surgery, \\ Aretaieion Hospital, Medical School, University of Athens, Athens 11528, Greece
}

Received August 30, 2020; Accepted December 17, 2020

DOI: $10.3892 / \mathrm{mco} .2020 .2202$

\begin{abstract}
Cystic lymphangiomas are uncommon benign lesions extremely rare in the adult population. Most cases are found in the neck and axillary regions; while $<1 \%$ of patients present with cystic lymphangiomas in the mesentery, greater omentum and retroperitoneum. The present report describes a rare case of large omental lymphangioma mimicking ovarian mass. A 40-year-old G2P2 female patient presented without symptoms for routine gynecological examination. Transvaginal ultrasound examination revealed a cystic mass with a maximum diameter of $10 \mathrm{~cm}$ localized at the right parametrium space, suggestive of large cystic lesion of the right ovary. Further preoperative evaluation by magnetic resonance imaging indicated that the mass was either cystic lymphangioma or mesenteric cyst. Complete excision of the cyst without need for gastrectomy was performed via laparotomy under general anaesthesia. Histology revealed omental lymphangioma. Most abdominal lymphangiomas are initially asymptomatic. The role of synchronous imaging examinations, such as ultrasonography and magnetic resonance imaging, in diagnosis of these lesions is crucial. Full preoperative differential diagnosis evaluation in cases of large intraabdominal lesions is required in order to decide the appropriate surgical approach and management.
\end{abstract}

\section{Introduction}

Cystic lymphangiomas are rare benign tumors that result from a developmental failure of lymphatic system or inflammation of lymphatic vessels causing obstruction and subsequent development of lymphangioma (1-3). These lesions usually appear in the head, neck and axillary regions, most often

Correspondence to: Dr Charalampos Grigoriadis, Department of Obstetrics and Gynecology, Leto Maternity Hospital, 7-13 Mouson Street, Athens 11524, Greece

E-mail: xarisgrigoriadis@yahoo.gr

Key words: abdominal cyst, lymphangioma, omentum, ovary, ultrasonography in children $(4,5)$. Less than $1 \%$ of patients present with cystic lymphangiomas in the mesentery, greater omentum and retroperitoneum $(1,3)$. Because of this low incidence and absence of symptoms in the majority of intraabdominal lymphangiomas, these tumors are often unexpectedly diagnosed in the operative field of other diseases $(6,7)$.

Most lymphangiomas into the abdominal cavity originate from the mesentery in which the majority of lymphatic channels is located. Therefore, intraabdominal lymphangiomas were previously named as 'mesenteric cystic lymphangiomas' (6). However, various locations and sizes of cystic lymphangiomas have been described. The greater and lesser omentum, as well as the retroperitoneum are other sites where cystic lymphangiomas can be found. Differential diagnosis between cystic lymphangiomas and mesenteric cysts that arise from mesothelial but not lymphatic tissue is of great importance (2). Lymphangiomas often behave aggressively, while mesenteric cysts do not. Histological examination after surgical excision is often needed to establish final diagnosis.

In this study, a very rare case of large omental cystic lymphangioma diagnosed in an adult woman during routine gynecological examination is described. The aim of this work is to underline the role of routine gynecological examination and the importance of full preoperative diagnostic evaluation in cases of large intraabdominal lesions in order to decide the appropriate surgical approach and management. The study has been reported in line with the SCARE criteria (8). Approval from Leto Maternity Hospital Ethics Committee (no. 42/2018) was given.

\section{Case report}

A 40-year-old G2P2 female patient presented, without symptoms, for routine gynecological examination. She had obstetrical history of two vaginal deliveries at term, no previous surgical interventions and medical history of Hashimoto's thyroiditis without medication.

Transvaginal ultrasound examination revealed a cystic mass, unilocular without solid components or papillary lesions (maximum diameter of $10 \mathrm{~cm}$ ) localized at the right parametrium space, suggestive of large cystic lesion of the right ovary (Fig. 1). Routine blood investigations as well as CA-125 marker were into 
normal ranges ( $29.4 \mathrm{U} / \mathrm{ml}$, n.r. $<35 \mathrm{U} / \mathrm{ml})$. Further preoperative evaluation withmagneticresonanceimaging(MRI)indicated that the mass was not arising from the right ovary. It was shown that it was either an intraabdominal cystic lymphangioma or mesenteric cyst.

Laparotomy, under general anesthesia, via midline vertical subumbilical incision was done, after obtaining informed consent from the patient. A large cystic mass originating from the greater omentum was detected. Complete excision of the lesion after dissection from the omentum, without need for gastrectomy, was performed. There was no need for frozen section as the macroscopic view of the specimen was not suggestive for malignancy.

Histology showed benign vascular cystic lesion, full of membranoid diaphragms, lymphatic vessels and smooth cells, with morphological signs suggestive of lymphangioma. Immunohistochemistry was positive for D2-40 marker, while negative for Calretinin and CK AE1/AE3. Two lymph nodes that were also removed were negative for malignant disease.

Post-operative period was uneventful. The patient was discharged on postoperative day 3 without complications. During this 2-year follow up period after initial surgery, no recurrence was identified.

Informed written consent was obtained from the patient for publication of this case report and accompanying images.

\section{Discussion}

The etiological factors and pathophysiological mechanisms that lead to lymphangiomas development remain unclear. As the vast majority of cases occur in children, the hypothesis that these lesions derive from a congenital abnormality of the lymphatic system is strongly supported (2). According to this pathophysiological route lymphangiomas may result from a developmental failure of the lymphatic system (lymphatic malformation). In our case the patient was not a child but a 40-year-old female patient without surgical history. So we have no evidence to identify the chronical presence of the tumor and its biological behavior, despite the fact that all previous transvaginal ultrasound examinations were normal.

In addition, our patient was asymptomatic as most patients with intraabdominal cystic lymphangiomas. This is the reason for which these masses are often diagnosed in the operative field of other diseases. However, symptoms including abdominal distension, pain, nausea and vomiting can be reported as the lesion progress (3). Potential complications of intraabdominal cystic lymphangiomas include infection, torsion, hemorrhage and small bowel obstruction (9).

Ultrasonography, computed tomography and magnetic resonance imaging are highly sensitive examinations that can be useful in preoperative differential diagnosis (10). Lymphangiomas are detected via ultrasonography as anechoic multilocular cystic masses with internal septa. In our case a large anechoic cystic mass was found via transvaginal ultrasonography into the right parametrium space in attachment with the uterus. This atypical localization of disease led us to a primary diagnosis of ovarian mass. However, magnetic resonance imaging revealed that right ovary was not the site from which this lesion was originating. A differential diagnosis

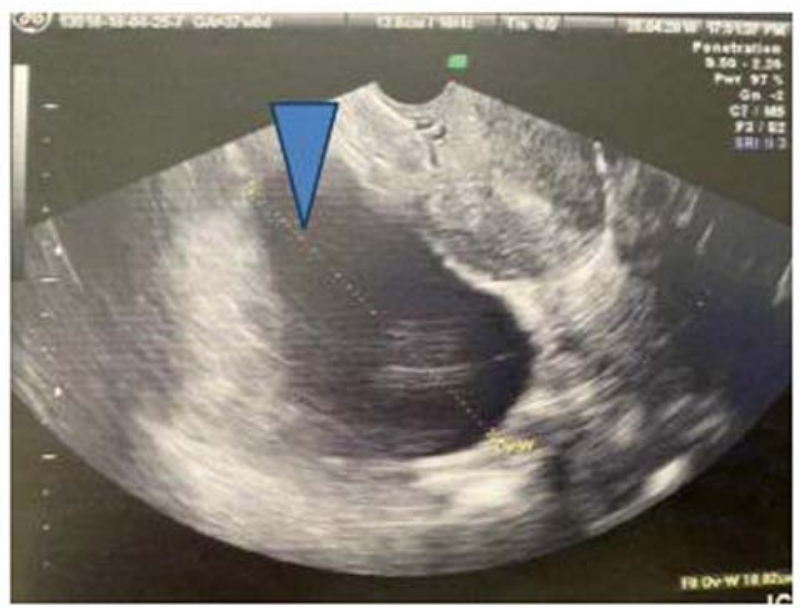

Figure 1. Transvaginal ultrasonographic appearance of a large cystic lesion (blue arrow) into the right parametrium in attachment with uterus.

problem between mesenteric cyst and cystic lymphangioma was observed after MRI as there are no specific radiological features to differentiate between these two lesions (2).

In such cases, like ours, pathological examination is needed in order to confirm diagnosis. Despite being difficult to differentiate via imaging examinations, these two lesions are histologically different. Lymphangiomas, as in our case, have an endothelial lining, foam cells and a wall full of lymphoid tissue, vessels with smooth muscles and lymphatic spaces, in contrast with mesenteric cysts which arise from mesothelial tissue.

Total resection of cystic lymphangiomas is considered as a useful treatment strategy in order to avoid life-threatening complications of these tumors which have the potential to grow and invade vital structures. Need for omentectomy or even gastrectomy has been reported for total removal of cystic lymphangiomas, depending on the tumors' characteristics, size and location (6). In our case the lesion was arising from the greater omentum near to the stomach, but after dissection, it was removed totally without need for further surgical intervention.

In conclusion, full preoperative differential diagnosis evaluation in cases of large intraabdominal lesions is needed in order to decide the appropriate surgical approach and management. Computed tomography and magnetic resonance imaging may add valuable information regarding parameters such as the size, location, other organ involvement, and solid components within cysts, that can contribute to preoperative surgical planning.

\section{Acknowledgements}

Not applicable.

\section{Funding}

No funding was received.

\section{Availability of data and materials}

The datasets used and/or analysed during the current study are available from the corresponding author on reasonable request. 


\section{Authors' contributions}

AT contributed to the study concept, design, data collection, data analysis and writing of the paper. CG was involved in data collection, data analysis and writing of the paper. KT was involved in study design and data analysis. IV developed the study concept and design. All authors read and approved the final manuscript.

\section{Ethics approval and consent to participate}

Approval from Leto Maternity Hospital Ethics Committee (no. 42/2018) was obtained.

\section{Patient consent for publication}

Written consent for publication was obtained from the patient.

\section{Competing interests}

The authors declare that they have no competing interests.

\section{References}

1. Hamaguchi Y, Arita S, Sugimoto N, Inamoto O, Takagi H, Kogire $M$ and Kitai T: Laparoscopic resection of abdominal cystic lymphangioma derived from lesser omentum: Case report. Medicine (Baltimore) 99: e18641, 2020.

2. Rao TN, Parvathi T and Suvarchala A: Omental lymphangioma in adults-rare presentation report of a case. Case Rep Surg 2012: 629482, 2012.
3. Losanoff JE, Richman BW, El-Sherif A, Rider KD and Jones JW: Mesenteric cystic lymphangioma. J Am Coll Surg 196: 598-603, 2003.

4. Kosir MA, Sonnino RE and Gauderer MW: Pediatric abdominal lymphangiomas: A plea for early recognition. J Pediatr Surg 26: 1309-1313, 1991.

5. Makni A, Chebbi F, Fetirich F, Ksantini R, Bedioui H Jouini M, Kacem M and Ben Safta Z: Surgical management of intra-abdominal cystic lymphangioma. Report of 20 cases. World J Surg 36: 1037-1043, 2012.

6. Kang BH, Hur H, Joung YS, Kim DK, Kim YB, Ahn CW, Han SU and Cho YK: Giant mesenteric cystic lymphangioma originating from the lesser omentum in the abdominal cavity. J Gastric Cancer 11: 243-247, 2011.

7. Takiff H, Calabria R, Yin L and Stabile BE: Mesenteric cysts and intra-abdominal cystic lymphangiomas. Arch Surg 120: 1266-1269, 1985

8. Agha RA, Borrelli MR, Farwana R, Koshy K, Fowler AJ and Orgill DP; SCARE Group: The SCARE 2018 statement: Updating consensus surgical CAse REport (SCARE) guidelines. Int J Surg 60: 132-136, 2018.

9. Christensen JA, Fuller JW, Hallock JA and Sherman RT: Mesenteric cysts: A cause of small bowel obstruction in children. Am Surg 41: 352-354, 1975.

10. Chou YH, Tiu CM, Lui WY and Chang T: Mesenteric and omental cysts: An ultrasonographic and clinical study of 15 patients. Gastrointestinal Radiol 16: 311-314, 1991.

This work is licensed under a Creative Commons Attribution-NonCommercial-NoDerivatives 4.0 International (CC BY-NC-ND 4.0) License. 